\title{
Trend of Complete Hydatidiform Mole
}

\author{
Thapa K, Shrestha $M,{ }^{1}$ Sharma $S,{ }^{2}$ Pandey $S^{1}$ \\ 'Paropakar Maternity and Women's Hospital, Thapathali, Kathmandu, ${ }^{2}$ Ministry of Health and Population Ramshahpath, \\ Kathmandu.
}

\section{ABSTRACT}

Introduction: Complete Hydatidiform mole is one of the most frequent abnormal pregnancies. This review studies the trend of complete mole in Paropakar Maternity and Women's hospital and clinical ability to detect it.

Methods: This is a retrospective study of 504 cases of complete hydatidiform mole recorded at Paropakar maternity and women's hospital, Kathmandu, during 2058-2065 B.S. Medical records were reviewed and incidence, clinical presentation and method of diagnosis were studied.

Results: During the study period, there were 139117 births and 504 complete moles, 12 partial moles, 48 persistent gestational tumours, six choriocarcinoma and four invasive moles recorded in the hospital. The incidence of complete mole was one per 276 births. It was prevalent among women younger than 29 years (80\%) and among the primigravidae (36.7\%). More than $90 \%$ women presented in the first half of their pregnancy and vaginal bleeding was the main complaint (68.3\%). Suction evacuation, dilation and evacuation followed by sharp curettage and abdominal hysterectomy were performed in $80.6 \%, 17.6 \%$ and $1.2 \%$ of the women respectively. Persistent mole and choriocarcinoma developed in $9.5 \%$ and $0.4 \%$ respectively.

Conclusions: Complete mole has the highest incidence. It affects mostly younger women and presents with vaginal bleeding most of the time, usually in the first half of their pregnancy.

Key Words: complete hydatidiform mole, gestational trophoblastic disease, persistent gestational tumours

\section{INTRODUCTION}

Gestational trophoblastic disease (GTD) is a group of neoplasms derived from placental trophoblastic tissue. It includes a spectrum of interrelated tumors including complete and partial hydatidiform mole, placental site trophoblastic tumor (PSTT) and choriocarcinoma. ${ }^{1}$
The incidence of GTD varies significantly across the world with 12.5 per 1000 births in Taiwan to 0.4 per 1000 births in the US. ${ }^{2}$ In Nepal, Maternity Hospital reported an incidence of 3.24 per 1000 deliveries in 1986-1987 and Tribhuwan University Teaching Hospital

Correspondence:

Dr. Kusum Thapa

Paropakar Maternity and Women's Hospital

Thapathali, Kathmandu, Nepal

Email: kusumthapa2006@hotmail.com

Phone: 9841555740 
reported 8.04 in 1985-1988. ${ }^{1,3}$ More recently in 2005, four different hospitals in Kathmandu valley recorded an incidence of GTD as 5.1, 2.9, 4, 2.8 per 1000 live births.

It commonly affects women in reproductive age group ${ }^{3,4}$ and presents with some typical symptoms, which helps in diagnosis. ${ }^{2}$ Recently, due to wide use of ultrasonography (USG) and serum в-hCG tests, molar pregnancy gets diagnosed quite early in the first trimester, even before any symptom appear. ${ }^{5}$

Thus, this study was conducted to see the recent trend in the incidence of molar pregnancy, including its signs and symptoms.

\section{METHODS}

This is a retrospective descriptive study conducted at Paropakar Maternity and Women's Hospital. It reviewed all the medical records since first April 2001 till April 2009. All the cases diagnosed and primarily treated as GTD were included in the study. The maternal age, gestational period at the time of presentation, gravidity, clinical presentation, diagnostic tools and status of previous pregnancy were noted for each of those cases. Information on histopathological reports and follow-up details were not available for all the cases, so these variables were not assessed in the study.

Those cases with hemoglobin level less than $7 \%$ were termed severely anemic, within 7 to $8.9 \%$ were termed moderately anemic and within $9 \%$ to $10.9 \%$ were termed mild anemic.

The study was ethically approved by the ethical research board of the hospital. Statistical analysis was done with Microsoft Excel 2007.

\section{RESULTS}

During the study period of eight years, the hospital recorded 139117 deliveries and 574 GTDs, which included 504 complete moles (CM), 12 partial moles (PM), 48 persistent gestational trophoblastic tumor (PGTT), six choriocarcinoma and four invasive moles.

The overall incidence of GTD was 4.1 per 1000 deliveries. Specifically, incidence of CM, PM, PGTT, choriocarcinoma and invasive mole were one per 276, 1 per 11593, one per 2898, one per 21386 and one per 34779 deliveries respectively.

By ethnicity, 54\% women were Mongolians. The age of women with CM ranged from 15 to 51 years with median of 22.9 years. More than $80 \%$ of them were below 29 years of age (Figure 1). Out of 504 women, 476 (94.4\%) presented to the hospital at or before $22^{\text {nd }}$ week of gestation (Figure 2). Majority of the women $(36.7 \%$ ) were primigravidae (Table 1). There were two cases who had a previous molar pregnancy.

\section{Table 1. Distribution by Gravidity}

\begin{tabular}{|c|c|c|}
\hline SN & Gravidity & No. of CM (\%) \\
\hline 1. & Primi gravida & $185(36.7)$ \\
\hline 2. & $2^{\text {nd }}$ gravida & $159(31.5)$ \\
\hline 3. & $3^{\text {rd }}$ gravida & $64(12.7)$ \\
\hline 4. & $4^{\text {th }}$ gravida & $42(8.3)$ \\
\hline 5 & $5^{\text {th }}$ gravida & $27(5.4)$ \\
\hline 6. & $6^{\text {th }} \quad$ gravida & $7(1.4)$ \\
\hline 7. & $7^{\text {th }} \quad$ gravida & $7(1.4)$ \\
\hline 8. & $8^{\text {th }} \quad$ gravida & $5(1.0)$ \\
\hline 9. & $9^{\text {th }} \quad$ gravida & $6(1.2)$ \\
\hline 10. & $10^{\text {th }}$ gravida & $2(0.4)$ \\
\hline Total & & $504(100)$ \\
\hline
\end{tabular}

The main complaints were vaginal bleeding (68.3\%), pain in the lower abdomen with vaginal bleeding $(21.4 \%)$, lower abdominal pain $(8.7 \%)$ and excessive vomiting $(0.8 \%)$. Four $(0.8 \%)$ patients were asymptomatic (Table 2). Besides these symptoms, majority of the women with molar pregnancy also had other complications of pregnancy, for which they were admitted. The major ones were threatened abortion $(61,12.1 \%)$, incomplete abortion $(34,6.7 \%)$, missed abortion $(8,1.6 \%)$, hyperemesis gravidarum $(5,1 \%)$ (Table 3 ).

Table 2. Symptoms at presentation

\begin{tabular}{lll}
\hline SN & Symptoms & No. of CM (\%) \\
\hline 1 & Vaginal bleeding & $344(68.3)$ \\
2 & Pain abdomen and vaginal bleeding & $108(21.4)$ \\
3 & Lower abdomen pain & $44(8.7)$ \\
4 & Excessive vomiting & $4(0.8)$ \\
5 & Haemoptysis & - \\
6 & Preeclampsia & - \\
7 & No symptoms & $4(0.8)$ \\
& Total & $504(100)$ \\
\hline
\end{tabular}

Among 504 women, 257(51\%) had uterine size larger than the size expected for the gestational period (Figure 3). Two hundred and ninety (57.5\%) women had mild to severe form of anemia. Thirty three $(11.3 \%)$ of them were severely anemic and three of them had hemoglobin level less than $4 \mathrm{gm} \%$.

As basic investigation, USG abdomen was done in $497(98.6 \%)$, chest $x$-ray in $377(74.8 \%)$ and only serum в hCG in $79(15.7 \%)$. As primary management, 413 $(81.9 \%)$ had suction evacuation and $90(17.9 \%)$ had cervical dilation and evacuation. One woman had profuse 
bleeding during evacuation of uterus corresponding to 36 weeks gestation, for which hysterectomy was done. Similarly, other five women had hysterectomy for persistent vaginal bleeding during follow-up period. Check dilation and curettage was done for $9(1.8 \%)$ complete moles. However, such procedure was not performed since 2004 onwards. During follow-up period of six months, $48(9.5 \%)$ developed PGTT and two $(0.4 \%)$ developed choriocarcinoma.

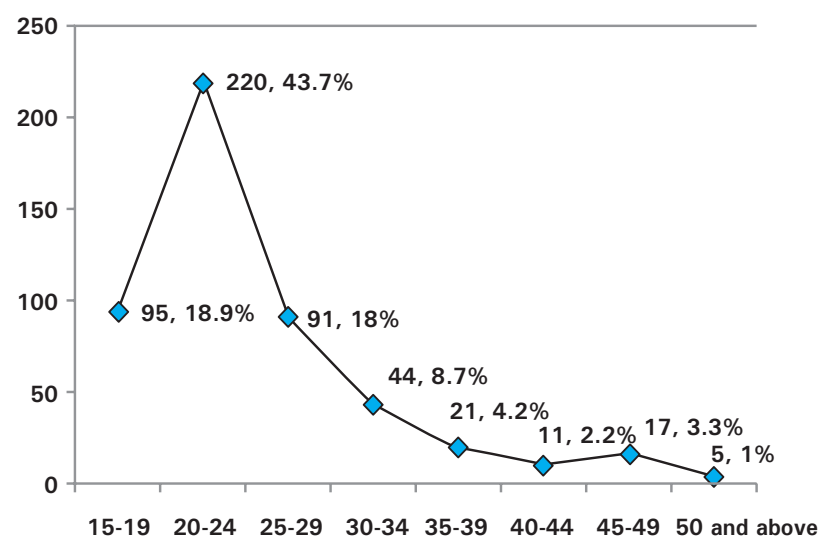

Figure 1. Age distribution of the cases

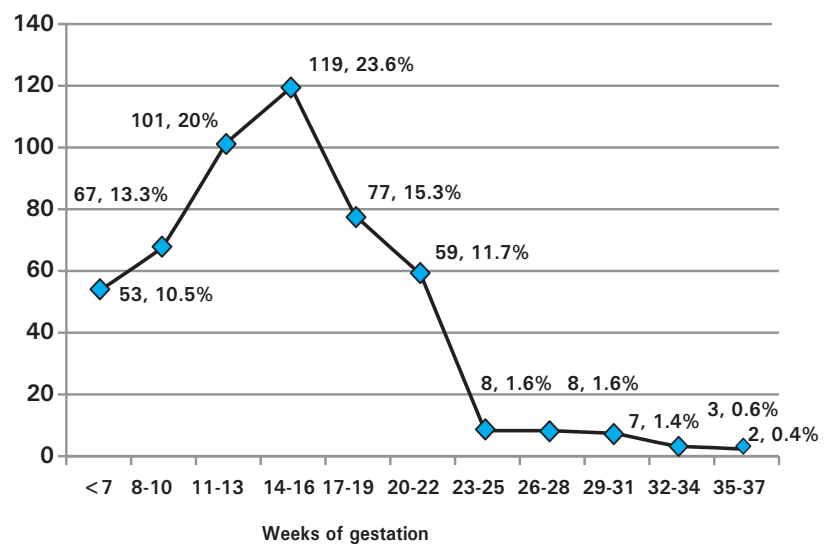

\section{Figure 2. Distribution by gestational age}

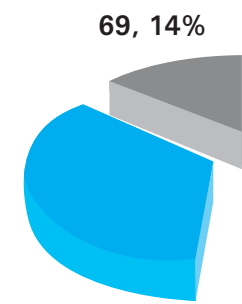

$178,35 \%$

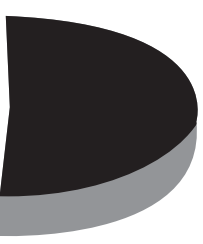

$257,51 \%$
Large for gestational age

Small for gestational age

Corresponds to gestational age
Figure 3. Uterine size
Table 3. Diagnosis on Admission

\begin{tabular}{ll}
\hline SN Diagnosis on admission & No. of CM (\%) \\
\hline 1. Threatened abortion & $61(12.1)$ \\
2. Incomplete abortion & $34(6.7)$ \\
3. Missed abortion & $8(1.6)$ \\
4. Hyperemesis gravidarum & $5(1.0)$ \\
5. Inevitable abortion & $4(0.8)$ \\
6. Blighted ovum & $4(0.8)$ \\
7. Ectopic pregnancy & $2(0.4)$ \\
8. APH & $1(0.2)$ \\
9. Fibroid uterus & $1(0.2)$ \\
10. Fibroid uterus with pregnancy & $1(0.2)$ \\
11. DUB & $1(0.2)$ \\
12. Molar pregnancy (USG) & $375(74.4)$ \\
13. Clinical diagnosis of molar pregnancy & $7(1.4)$ \\
\hline
\end{tabular}

\section{DISCUSSION}

The study reported an incidence of GTD as 4.1 per 1000 deliveries. Previous studies in different hospitals of Nepal reported a varying figure ranging from 2.8 to 5.1 per 1000 deliveries. ${ }^{1}$ The same figure is 0.4 in the US. ${ }^{2}$ Likewise, the incidence of CM in the study was one per 276, which is higher than the incidence of 1 per 676 reported by a study at King Fahad Hospital, Saudi Arabia. ${ }^{5}$ Our study also found GTD more common among mongoloids (54\%), contrast to Acharya $\mathrm{G}^{3}$ who found no difference in the incidence in GTD among patients of different ethnic groups in his study conducted in the year 1996.

Our study found high prevalence of molar pregnancy among women aged 29 and below (80\%). Survey among Nigerian women found that those aged over 30 years, multiparous, particularly over parity four had higher risk of having molar pregnancy. ${ }^{6}$ King Fahad Hospital of Saudi Arabia also found increased risk of complete mole among both teenagers and women above 30 years. ${ }^{5}$ In addition, Berkowitz, too reported that maternal age above 35 years is a risk factor for developing CM. ${ }^{7}$ Likewise, Acharya ${ }^{3}$ observed $87.5 \%$ of molar pregnancy among young women below 35 years. One study also found increased risk of GTD among women between 20-29 years of age. ${ }^{2}$ Thus, the women in their twenties and thirties should be watched out for molar pregnancy during their ante natal checkups.

Our study found a higher incidence of mole among primigravidae $(36.7 \%)$. Many studies claimed that there is no clear relation between gravidity, parity and GTD. ${ }^{4,8}$

However study from Nigeria reported that parity four and above in particular adds to the risk of GTD. ${ }^{6}$ 
It has been reported that women who had had previous molar pregnancy, appear to have a risk of $1-2 \%$ of having another molar pregnancy. ${ }^{1,7}$ Our study found two such cases $(0.4 \%)$. But a study at Patan hospital reported none of the molar pregnancy patients had a previous history of molar pregnancy. ${ }^{3}$

Classical clinical and pathologic features of molar pregnancy have been well known for many years. Vaginal bleeding, uterine size large for date, anaemia, early onset preeclampsia, hyperemesis gravidarum and theca lutein cyst are common clinical manifestations, and grapelike swollen villi (vesicles) and diffuse hyperplasia of trophoblasts without fetus are cardinal pathologic features of complete Hydatidform mole. ${ }^{9}$ Our study also found vaginal bleeding and lower abdominal pain as the most frequent symptoms among the patients. There have been reports of vaginal bleeding varying from spotting to profuse bleeding occurring intermittently for weeks and even months. ${ }^{8}$ Chhabra and Sinha reported $97 \%$ of women with complete mole had vaginal bleeding. ${ }^{2}$ We found this complaint among $68.3 \%$ of the women.

Literature reports excessive vomiting as a common symptom occurring among $14 \%$ of complete mole patients. ${ }^{4}$ However, our study found only four women $(0.8 \%)$ with such problem, and so did another study in Patan Hospital, Kathmandu. ${ }^{3}$ May be the patients presented quite early before their symptoms got worse.

Apart from the classical presentation of molar pregnancy, it could present with some other conditions as well, which are precipitated by molar pregnancy like preeclampsia, abortion. Literature supports that preeclampsia before 24 weeks of gestation may be due to $\mathrm{CHM}^{8}{ }^{8}$ Our study didn't find any case of preeclampsia, but there were $107(21.2 \%)$ abortive cases.

Usually, uterus is large for date in about $50 \%$ of molar pregnancies. ${ }^{8}$ Chhabra and Sinha ${ }^{2}$ and Acharya ${ }^{3}$ found it true among $75 \%, 81.2 \%$ of molar pregnancies respectively. Our review showed 257 (51\%) of women had uterine enlargement beyond their gestational age. In present scenario with better and wide availability of modern investigations, molar pregnancy could be picked up early and thus, the uterus larger for date could be less frequently encountered. This might be the reason, Felembam et al reported only $28 \%$ of their cases had uterus large for date. ${ }^{5}$ The early diagnostic tool could be an abdominal ultrasonography, and $\operatorname{Rajan}^{10}$ recommended routine abdominal USG after eight weeks of pregnancy to detect mole earlier than waiting for complications.

Our study found anemia among $57.5 \%$ of women and $50 \%$ required blood transfusion. Profuse vaginal bleeding could be the cause. But, anemia was found among only $15.5 \%$ in the study conducted at King Fahad Hospital. ${ }^{5}$ This could be due to early diagnosis of molar pregnancy, as literature also supports that early diagnosis of Hytadiform mole in first trimester reduce prevalence of anemia from $54 \%$ to $5 \%{ }^{4}$

Suction evacuation is considered the gold standard procedure to terminate molar pregnancy. ${ }^{1}$ In our study, $81.9 \%$ women with mole had suction evacuation. However; one woman underwent hysterectomy for profuse hemorrhage while performing evacuation of 36week size uterus. Five other had hysterectomy for persistent vaginal bleeding during follow-ups, the cause for vaginal bleeding was not well documented.

Malignant transformation is a dreaded complication of molar pregnancy and thus molar pregnancy requires regular follow-up. In our study, two women $(0.4 \%)$ developed choriocarcinoma during the follow-up period of six months. Recent studies have shown that prophylactic hysterectomy does not reduce incidence of malignant transformation. ${ }^{2,3}$

\section{CONCLUSIONS}

Complete mole is not only the most common GTD, but also the most common antecedent condition to PGTTs. Age, parity, gestational age could have causal relationship with molar pregnancies. Vaginal bleeding and lower abdominal pain are the most common clinical features. Molar pregnancy should be cautiously investigated for among patients with such features.

\section{REFERENCES}

1) NESOG. Guideline of management of Gestational Trophoblastic disease. Kathmandu: NESOG; 2009.

2) Chhabra A, Sinha P. Gestational Trophoblastic disease - some observation. The Journal of obstetrics and Gynecology of India. 1988;38:590-3.

3) Acharya G. Gestational Trophoblastic Disease in Nepal. J Nepal Med Assoc. 1996;34:226-35.

4) Seckl JM, Newlands ES. Gestational Trophoblastic Tumours. In: Shaw RW, Soutter WP, Stanton SL, editors. Gynecology. 3rd ed. Churchill Livingstone; 2003. p. 653-64.

5) Felembam AA, Bakri YN, Alkharif HA, Altuwaijri SM, et al. Complete Molar Pregnancy. Am J The journal of reproductive medicine.1998;43:11-3.

6) Ekpo MD. Hydatidiform Mole in Nigeria. Journal of Obstetrics and Gynecology. 1990;10:363-6.

7) Berkowitz RS, ImSamuel S, Bernstein MR, Goldstein DP. Gestational Trophoblastic Disease. Am J The Journal of Reproductive Medicine. 1998;43:81-6.

8) Burger RA, Creasman WT. Gestational Trophoblastic Neoplasia. In: Disaia PJ, Creasman WT, editors. Clinical Gynecologic Oncology. 6th ed. Mosby; 2002. p. 185-210.

9) Mosher R, Goldstein PD, Berkowitz R, Bernstein M, and Genest DR. Complete Hydatidiform Mole. Am J the Journal of Reproductive Medicine.1998;43:21-7.

10) Rajan R. Gestational Trophoblastic Neoplasia - Certain Interesting Observation. The Journal of Obstetrics and Gynecology of India. 1988;38:73-7. 